Portland State University

PDXScholar

Electrical and Computer Engineering Faculty

Publications and Presentations

Electrical and Computer Engineering

6-1-1991

\title{
Pump polarization effects in cw dye lasers
}

Lee W. Casperson

Portland State University

W. J. Sandle

A. C. Wilson

D. M. Warrington

R. J. Ballagh

Follow this and additional works at: https://pdxscholar.library.pdx.edu/ece_fac

Part of the Electrical and Computer Engineering Commons

Let us know how access to this document benefits you.

\section{Citation Details}

Casperson, L. W., Sandle, W. J., Wilson, A. C., Warrington, D. M., \& Ballagh, R. J. (1991). Pump polarization effects in cw dye lasers. Journal Of Applied Physics, 69(12), 8005.

This Article is brought to you for free and open access. It has been accepted for inclusion in Electrical and Computer Engineering Faculty Publications and Presentations by an authorized administrator of PDXScholar. Please contact us if we can make this document more accessible: pdxscholar@pdx.edu. 


\title{
Pump polarization effects in cw dye lasers
}

\author{
Lee W. Casperson, a) W. J. Sandle, A. C. Wilson, D. M. Warrington, and R. J. Ballagh \\ Department of Physics, University of Otago, P. O. Box 56, Dunedin, New Zealand
}

(Received 11 December 1990; accepted for publication 22 February 1991)

The effects of pump polarization on the performance of $\mathrm{cw}$ dye lasers have been investigated.

A recently developed semiclassical model for synchronously pumped mode-locked dye

lasers is adapted to the problem of cw dye-laser oscillation, and analytic expressions are obtained for the relationship between the pump power, pump polarization, and dye-laser output power. The theoretical results are compared with experimental data obtained using an argon-laser-pumped rhodamine $6 \mathrm{G}$ dye laser.

\section{INTRODUCTION}

Optically pumped lasers can operate with unpolarized pumping mechanisms such as flashlamps or incandescent filaments, or they may be pumped by polarized light sources such as other lasers. Dye lasers, in particular, are often designed to use other lasers as their pump sources. The pump lasers are typically linearly polarized, which tends to favor the gain for one signal polarization. The polarization characteristics of the spontaneous and stimulated emission from a dye-laser medium have been of interest since the earlicst pulsed dye-laser studies, ${ }^{1}$ and several polarization investigations of such pulsed systems have been performed..$^{2-6}$ The stimulated emission studies have emphasized the output polarization from otherwise isotropic dye-lasers oscillators. However, most practical dye lasers are based on anisotropic cavities incorporating Brewster surfaces, and the characteristics of these systems as a function of pump polarization are also of interest. The polarization characteristics of $\mathrm{cw}$ dye-laser amplifiers and oscillators have also been studied. ${ }^{7-10}$ Because of their wide frequency tuning range and potentially very high frequency stability, cw dye lasers are now used extensively in highresolution spectroscopic studies. In spite of the versatility of dye lasers and other similarly operated lasers, however, there remain fundamental aspects of the operation of these systems that are not yet thoroughly understood.

Recently, the polarization characteristics of synchronously pumped mode-locked dye lasers have also been studied, and a semiclassical model was developed which is able to explain several aspects of the mode-locked laser's performance in terms of the polarization of the input pump-laser beam. ${ }^{11}$ As in most practical dye lasers, the model assumed that the dye molecules have an isotropic orientational distribution. The results of that modeling effort were found to be in good agreement with experimental data obtained using a synchronously pumped mode-locked rhodamine $6 \mathrm{G}$ laser pumped by an argon-ion laser. At an intermediate stage in the development of the model, the formalism also provides a reasonably general description of cw operation of dye-laser oscillators. The purpose of this study is to focus more closely on these cw implications. The main result is a closed-form relationship between the pump power, pump polarization, and dye-laser intensity. While there have been several previous discussions of polarization in $\mathrm{cw}$ dye lasers, solutions have not been given for the important problem of the saturated intensity in laser oscillators having isotropic orientational distributions.

In Sec. II a semiclassical laser model is applied to the analysis of a cw dye-laser oscillator in which the pump field propagates collinearly with the signal field, but is polarized at an arbitrary angle. The principal results of this analysis are detailed solutions for the dye-laser intensity. Experimental results are compared with the theory in Sec. III.

\section{THEORY}

The energy-level model used here is the same four-level system used in other dye-laser studies. ${ }^{8,9,11}$ The pump absorption occurs between level 0 and level 3 (Fig. 1 of Ref. 11 ), while the signal stimulated emission takes place between levels 2 and 1 . The molecules in level 3 decay nonradiatively to level 2 , and molecules in the lower laser level have a vibrational relaxation time of $\tau_{1}$ for transitions to the ground state. The spontaneous decay time for the laser transition is represented by $\tau_{2}$. We assume that the electric dipoles associated with the pump and signal transitions are parallel and in a direction fixed by the geometry of the molecules, and that the dye molecules are isotropically distributed in the fluid. The polarizations of the signal and pump fields are assumed to be linear with an angular separation of $\alpha$, and we denote by $\theta$ the angle between the signal-field polarization and the signal-field dipole $\mu_{s}$ of a given molecule ( $\mathrm{sec}$ Fig. 1). The starting equations for the present analysis can be adopted directly from Ref. 11 . The principal restrictions include near-line-center operation, collinear pumping, negligible saturation or coherence effects on the pump transition, and negligible intersystem crossing or orientational relaxation. Most of these restrictions are appropriate for most $\mathrm{cw}$ dye-laser oscillators. In our own experiments based on a liquid solution of rhodamine $6 \mathrm{G}$ molecules, the assumption of negligible intersystem crossing or orientational relaxation will be shown to allow only a qualitative comparison with the theory in its present form.

\footnotetext{
'Permanent address: Department of Electrical Engineering, Portland State University, P.O. Box 751, Portland, OR 97207-0751.
} 




FIG. 1. Relative orientations of the pump and signal electric fields and the signal dipole moment of a given molecule. The pump dipole moment is assumed parallel to the signal dipole.

Choosing the plane-wave fields to propagate in the $z$ direction with velocity $v$ and putting $x=\cos \theta$, the normalized forms of the semiclassical laser equations are given by Eqs. (23)-(26) of Ref. 11:

$$
\begin{aligned}
\frac{\partial D(x, z, t)}{\partial t}= & -\frac{1}{\tau_{2}}\left\{\left(1+\frac{\tau_{2}}{2 \tau_{1}}\right) D(x, z, t)+\left(1-\frac{\tau_{2}}{2 \tau_{1}}\right)\right. \\
& \times M(x, z, t)+2 Q(x, z, t) A(z, t) x-P(z, t) \\
& \left.\times\left[x^{2}\left(1-\frac{3 \sin ^{2} \alpha}{2}\right)+\frac{\sin ^{2} \alpha}{2}\right]\right\},
\end{aligned}
$$

$$
\begin{aligned}
\frac{\partial M(x, z, t)}{\partial t}= & -\frac{1}{\tau_{2}}\left\{-\frac{\tau_{2}}{2 \tau_{1}} D(x, z, t)+\frac{\tau_{2}}{2 \tau_{1}} M(x, z, t)\right. \\
& \left.-P(z, t)\left[x^{2}\left(1-\frac{3 \sin ^{2} \alpha}{2}\right)+\frac{\sin ^{2} \alpha}{2}\right]\right\},
\end{aligned}
$$

$\frac{\partial Q(x, z, t)}{\partial t}=-\frac{1}{T_{s}}[Q(x, z, t)-A(z, t) D(x, z, t) x]$,

$\frac{\partial A(z, t)}{\partial z}+\frac{1}{v} \frac{\partial A(z, t)}{\partial t}=-\frac{\gamma_{s}}{2}\left(A(z, t)-\int_{0}^{1} Q(x, z, t) x d x\right)$.

The quantity $A(z, t)$ represents the signal electric field, in units of $\left(\hbar /\left|\mu_{s}\right|\right)\left(2 / \tau_{s} T_{s}\right)^{1 / 2}$. The remaining normalized variables are the pump rate $P(z, t)$, the population difference between the upper and lower levels of the laser transition $D(x, z, t)$, the population sum $M(x, z, t)$, and the molecular polarization $Q(x, z, t)$.

In Ref. 11 the equations corresponding to Eqs. (1)(4) above were adapted to the problem of a synchronously pumped mode-locked dye laser, and numerical solutions provided good agreement with a wide range of experimental measurements. In the present study Eqs. (1)-(4) will be adapted to the problem of $\mathrm{cw}$ laser oscillation. First, it may be noted that if there are no significant variations of the dependent variables within the coherence time $T_{s}$, the derivative may be set to zero in Eq. (3), and the polarization is given by

$$
Q(x, z, t)=A(z, t) D(x, z, t) x .
$$

With this substitution, Eqs. (1), (2), and (4) are replaced by

$$
\begin{aligned}
\frac{\partial D(x, z, t)}{\partial t}= & -\frac{1}{\tau_{2}}\left\{\left(1+\frac{\tau_{2}}{2 \tau_{1}}\right) D(x, z, t)\right. \\
& +\left(1-\frac{\tau_{2}}{2 \tau_{1}}\right) M(x, z, t)+2 I(z, t) D(x, z, t) x^{2} \\
& \left.-P(z, t)\left[x^{2}\left(1-\frac{3 \sin ^{2} \alpha}{2}\right)+\frac{\sin ^{2} \alpha}{2}\right]\right\}
\end{aligned}
$$

$$
\begin{aligned}
\frac{\partial M(x, z, t)}{\partial t}= & -\frac{1}{\tau_{2}}\left\{-\frac{\tau_{2}}{2 \tau_{1}} D(x, z, t)+\frac{\tau_{2}}{2 \tau_{1}} M(x, z, t)\right. \\
& \left.-P(z, t)\left[x^{2}\left(1-\frac{3 \sin ^{2} \alpha}{2}\right)+\frac{\sin ^{2} \alpha}{2}\right]\right\},
\end{aligned}
$$

$$
\begin{aligned}
& \frac{\partial I(z, t)}{\partial z}+\frac{1}{v} \frac{\partial I(z, t)}{\partial t} \\
& =-\gamma_{s} I(z, t)\left(1-\int_{0}^{1} D(x, z, t) x^{2} d x\right),
\end{aligned}
$$

where $I(z, t)=A^{2}(z, t)$ is the normalized intensity. In this form the governing equations might be called rate equations because they govern the population densities and intensities while neglecting possible phase differences between the molecular polarization and the field. On the other hand, bccause of their semiclassical ancestry, these equations can account rigorously for the isotropic orientational distribution of the dye molecules and a possible nonzero angle between the pump and signal-field polarizations.

In a steady-state laser amplifier, all time dependencies go away and Eqs. (6) -(8) become

$$
\begin{aligned}
& 0=\left(1+\frac{\tau_{2}}{2 \tau_{1}}\right) D(x, z) \\
& +\left(1-\frac{\tau_{2}}{2 \tau_{1}}\right) M(x, z)+2 I(z) D(x, z) x^{2} \\
& -P(z)\left[\dot{x}^{2}\left(1-\frac{3 \sin ^{2} \alpha}{2}\right)+\frac{\sin ^{2} \alpha}{2}\right], \\
& 0=-\frac{\tau_{2}}{2 \tau_{1}} D(x, z)+\frac{\tau_{2}}{2 \tau_{1}} M(x, z) \\
& -P(z)\left[x^{2}\left(1-\frac{3 \sin ^{2} \alpha}{2}\right)+\frac{\sin ^{2} \alpha}{2}\right], \\
& \frac{d I(z)}{d z}=-\gamma_{s} I(z)\left(1-\int_{0}^{1} D(x, z) x^{2} d x\right) .
\end{aligned}
$$

From Eq. (10) the population sum is given by 
$M(x, z)=D(x, z)+\frac{2 \tau_{1}}{\tau_{2}} P(z)\left[x^{2}\left(1-\frac{3 \sin ^{2} \alpha}{2}\right)+\frac{\sin ^{2} \alpha}{2}\right]$

With this substitution Eq. (9) reduces to

$$
\begin{aligned}
0= & D(x, z)+I(z) D(x, z) x^{2}-\left(1-\frac{\tau_{1}}{\tau_{2}}\right) \\
& \times P(z)\left[x^{2}\left(1-\frac{3 \sin ^{2} \alpha}{2}\right)+\frac{\sin ^{2} \alpha}{2}\right] .
\end{aligned}
$$

From Eq. (13) the population difference is given by

$$
\begin{aligned}
D(x, z)= & \left(1-\frac{\tau_{1}}{\tau_{2}}\right) P(z)\left[x^{2}\left(1-\frac{3 \sin ^{2} \alpha}{2}\right)+\frac{\sin ^{2} \alpha}{2}\right] \\
& \times\left[1+I(z) x^{2}\right]^{-1 .}
\end{aligned}
$$

With this substitution into Eq. (11), the dye-laser amplifier is governed by the single equation

$$
\begin{aligned}
\frac{d I(z)}{d z}= & -\gamma_{s} I(z)\left\{1-\left(1-\frac{\tau_{1}}{\tau_{2}}\right) P(z)\right. \\
& \times \int_{0}^{1}\left[x^{2}\left(1-\frac{3 \sin ^{2} \alpha}{2}\right)+\frac{\sin ^{2} \alpha}{2}\right] \\
& \times\left[1+I(z) x^{2}\right]^{-1} x^{2} d x
\end{aligned}
$$

Equation (15) can be applied to calculate the output characteristics of a dye-laser oscillator.There are several ways in which this can be done, depending on the oscillator configuration; and some techniques for including high gain per pass, standing-wave effects, and transverse effects are described in Ref. 12. For the simplest one-dimensional ring-laser case with low signal gain and little pump absorption per pass, it follows from Eq. (15) that the increase in intensity after one round trip can be written

$$
\begin{aligned}
\Delta I= & \gamma_{s} I I P\left(1-\frac{\tau_{1}}{\tau_{2}}\right) \int_{0}^{1}\left[x^{2}\left(1-\frac{3 \sin ^{2} \alpha}{2}\right)+\frac{\sin ^{2} \alpha}{2}\right] \\
& \times\left(1+I x^{2}\right)^{-1} x^{2} d x-\gamma_{s} I I,
\end{aligned}
$$

where $l$ is the length of the amplifying medium. On the other hand, the mirror loss after one round trip is

$$
\Delta I=(1-R) I,
$$

where $R$ is the reflectivity of the output mirror. If the laser cavity has other lossy elements, further terms proportional to $I$ should be added to the right-hand side of Eq. (17).For stable oscillation the round-trip gain must equal the roundtrip loss, and so the left-hand sides of Eqs. (16) and (17) are equal. The resulting oscillator equation can be written

$$
\begin{aligned}
1= & \frac{\gamma_{s} l P}{(1-R)+\gamma_{s} l}\left(1-\frac{\tau_{1}}{\tau_{2}}\right) \int_{0}^{1}\left[x^{2}\left(1-\frac{3 \sin ^{2} \alpha}{2}\right)\right. \\
& \left.+\frac{\sin ^{2} \alpha}{2}\right]\left[1+\left[x^{2}\right]^{-1} x^{2} d x .\right.
\end{aligned}
$$

This result can be interpreted as an implicit equation for the intracavity intensity in terms of the pump rate $P$, pump angle $\alpha$, and other parameters of the laser medium and cavity.

It is usually simplest and most convenient to express laser intensity formulas in terms of a parameter which references the pump rate $P$ to its value $P_{\text {th }}$ at the oscillation threshold. We will define threshold to be the condition at which the intensity $I$ is zero for parallel alignment of the pump and signal fields $(\alpha=0)$. Thus, at threshold, Eq. (18) reduces to

$$
\begin{aligned}
1 & =\frac{\gamma_{s} l P_{\mathrm{th}}}{(1-R)+\gamma_{s} l}\left(1-\frac{\tau_{1}}{\tau_{2}}\right) \int_{0}^{1} x^{4} d x \\
& =\frac{\gamma_{s} l P_{\mathrm{th}}}{5\left[(1-R)+\gamma_{s} l\right]}\left(1-\frac{\tau_{1}}{\tau_{2}}\right) .
\end{aligned}
$$

If the threshold parameter is defined as $r=P / P_{\mathrm{th}}$, Eqs. (18) and (19) may be combined to obtain

$$
1=5 r \int_{0}^{1}\left[x^{2}\left(1-\frac{3 \sin ^{2} \alpha}{2}\right)+\frac{\sin ^{2} \alpha}{2}\right]\left(1+I x^{2}\right)^{-1} x^{2} d x .
$$

The necessary angle integrals can be more clearly isolated by writing Eq. (20) in the form

$$
\frac{1}{5 r}=\left(1-\frac{3 \sin ^{2} \alpha}{2}\right) J_{1}+\frac{\sin ^{2} \alpha}{2} J_{2},
$$

where the angle intcgrals are

$$
\begin{aligned}
& J_{1}=\int_{0}^{1} \frac{x^{4}}{1+I x^{2}} d x=\frac{1}{3 I}-\frac{1}{I^{2}}+\frac{1}{I^{5 / 2}} \tan ^{-1}\left(I^{1 / 2}\right) \\
& J_{2}=\int_{0}^{1} \frac{x^{2}}{1+I x^{2}} d x=\frac{1}{I}-\frac{1}{I^{3 / 2}} \tan ^{-1}\left(I^{1 / 2}\right)
\end{aligned}
$$

Equations (21)-(23) may now be combined into a single equation relating the three parameters $r, \alpha$, and $I$. It is not possible to solve this equation explicitly for the intensity $I$, and we find it most convenient to solve instead for the polarization alignment angle $\alpha$. It follows from Eq. (21) that the angle-dependent terms can be isolated in the form

$$
\frac{\sin ^{2} \alpha}{2}=\frac{1-5 r J_{1}}{5 r\left(J_{2}-3 J_{1}\right)},
$$

and thus the angle $\alpha$ is given by

$$
\alpha=\sin ^{-1}\left[\left(\frac{2-10 r J_{1}}{5 r\left(J_{2}-3 J_{1}\right)}\right)^{1 / 2}\right] \text {. }
$$

Equation (25) is plotted in polar form in Fig. 2 for several values of the threshold parameter $r$. The distance from the origin to a point on one of the curves is a measure of the laser intensity $I$ for those particular values of $r$ and alignment angle $\alpha$. It is clear from the figure that for small positive values of $r-1$ the laser will be below threshold when the pump polarization tends to be perpendicular to 


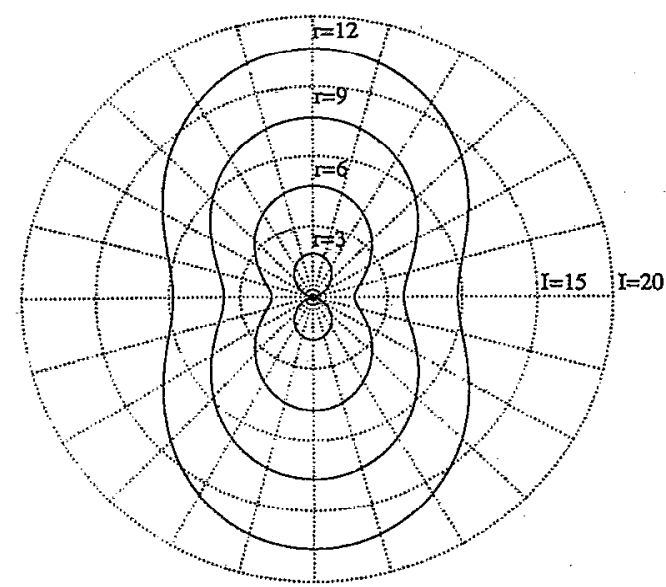

FIG. 2. Polar plol of dye-laser signal intensity $I$ as a function of the alignment angle $\alpha$ between the pump and signal fields for various values of the threshold parameter $r$. In this polar representation the intensity is the distance from the origin to a curve, and the vertical or long axis represents the signal polarization direction shown in Fig. 1 .

the signal polarization. For large positive values of $r-1$, on the other hand, strong lasing is obtained for all pump polarization angles.

The two specific pump polarization angles of greatest interest are $\alpha=0$, when the pump and signal are exactly parallel, and $\alpha=90^{\circ}$, when the pump and signal are exactly perpendicular. From Eq. (21) one finds in these two limits

$$
\frac{1}{5 r}= \begin{cases}J_{1}, & \text { parallel } \\ \left(J_{2}-J_{1}\right) / 2, & \text { perpendicular. }\end{cases}
$$

The two curves represented by Eq. (26) are plotted as solid lines in Fig. 3. One finds from the figure that oscillation

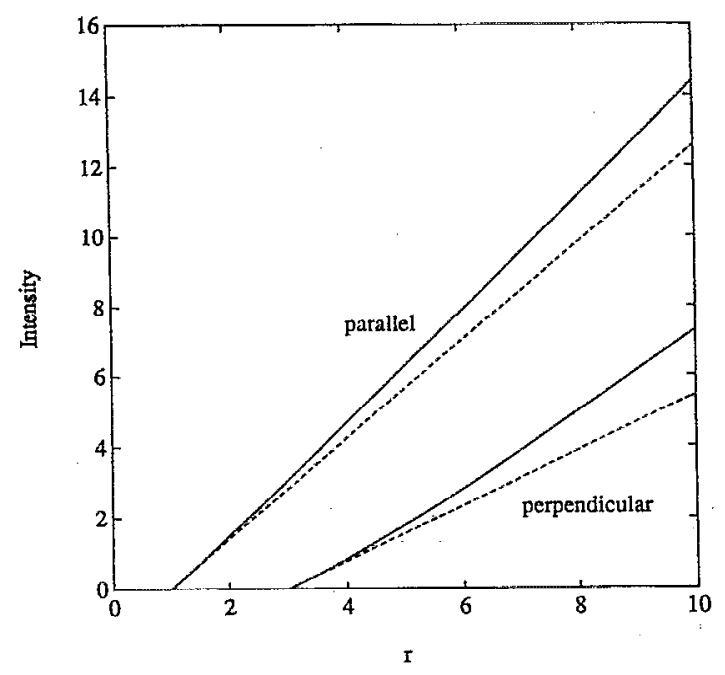

FIG. 3. Signal intensity $I$ as a function of the threshold parameter $r$ for the pump field parallel and perpendicular to the signal field. The solid lines are exact solutions of the closed form results given in Eqs. (28), and the dashed lines are the explicit approximations given in Eqs. (35). seems to be possible for the perpendicular pumping configuration only if the threshold parameter has the value $r=3$ or larger, and this conclusion will be confirmed analytically below.

In view of the nearly linear form of the curves in Fig. 3, it is of interest to see if useful simplifications of Eqs. (21) or (26) might be possible. For small intensities the angle integrals can in fact be represented by the simple powerseries expansions

$$
\begin{aligned}
& J_{1}=\frac{1}{5}-\frac{1}{7} I+\frac{1}{9} I^{2}-\frac{1}{11} I^{3}+\cdots, \\
& J_{2}=\frac{1}{3}-\frac{1}{5} I+\frac{1}{7} I^{2}-\frac{1}{9} I^{3}+\cdots .
\end{aligned}
$$

Therefore, Eq. (21) becomes

$$
\begin{aligned}
\frac{1}{5 r}= & \left(1-\frac{3 \sin ^{2} \alpha}{2}\right)\left(\frac{1}{5}-\frac{1}{7} I+\frac{1}{9} I^{2}-\frac{1}{11} I^{3}+\cdots\right) \\
& +\frac{\sin ^{2} \alpha}{2}\left(\frac{1}{3}-\frac{1}{5} I+\frac{1}{7} I^{2}-\frac{1}{9} I^{3}+\cdots\right) .
\end{aligned}
$$

To first order in the intensity, this equation reduces to

$$
\frac{1}{r}=\left(1-\frac{2 \sin ^{2} \alpha}{3}\right)-\left(\frac{5}{7}-\frac{4 \sin ^{2} \alpha}{7}\right) I \text {. }
$$

Equation (30) may be inverted, and again keeping only first-order terms in $I$, one obtains

$$
r=\frac{1}{1-2 \sin ^{2} \alpha / 3}\left(1+\frac{5 / 7-4 \sin ^{2} \alpha / 7}{1-2 \sin ^{2} \alpha / 3} I\right) .
$$

This low-intensity formula may be solved explicitly for the intensity, and the result is

$$
I=\frac{7\left(1-2 \sin ^{2} \alpha / 3\right)^{2}}{5\left(1-4 \sin ^{2} \alpha / 5\right)}\left(r-\frac{1}{1-2 \sin ^{2} \alpha / 3}\right) .
$$

In the limits of parallel and perpendicular pump orientation, Eq. (32) reduces to

$$
I= \begin{cases}\frac{7}{5}(r-1), & \text { parallel, } \\ \frac{7}{6}(r-3), & \text { perpendicular. }\end{cases}
$$

It is clear from this result that, as suggested by Fig, 3, the oscillation threshold for the perpendicular pump orientation is exactly three times higher than for the parallel orientation. These formulas are plotted as dashed lines in Fig. 3. For some purposes such simplified results might be adequate for comparison with experimental data.

For completeness one may also consider the limit of operation at high intensity far above the laser threshold. It follows from Eqs. (22) and (23) that in this limit $J_{1}$ reduces to $(3 I)^{-1}$ and $J_{2}$ reduces to $I^{-1}$. Now Eq. (21) can be solved explicitly for the intensity and the result is

$$
I=5 r / 3 \text {. }
$$

For this limit the laser intensity is independent of the polarization angle $\alpha$.

As a final point, it may be observed that the procedure developed here can be readily extended to other orientational distributions of the dye molecules. In the simplest case the molecules have a unidirectional distribution par- 


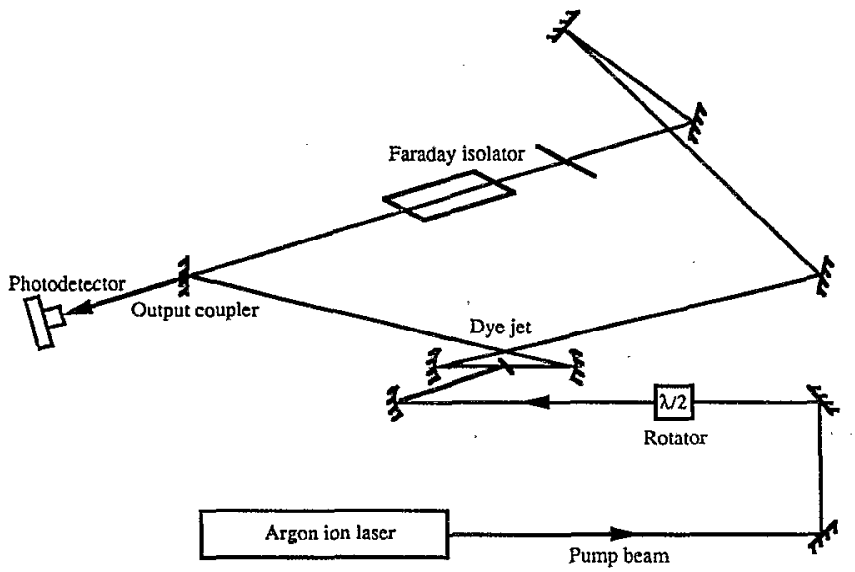

FIG. 4. Experimental unidirectional ring dye laser for pump polarization studies.

allel to the signal-field polarization. In this limit the angle variable $x=\cos \theta$ may be set to unity and the integral omitted in Eq. (18). Then one finds that Eq. (21) may be replaced by

$\frac{1}{r}=\left(1-\frac{3 \sin ^{2} \alpha}{2}\right) \frac{1}{1+I}+\frac{\sin ^{2} \alpha}{2} \frac{1}{1+I}=\frac{\cos ^{2} \alpha}{1+I}$.

Thus, the intensity for the unidirectional distribution is given by the simple formula

$$
I=r \cos ^{2} \alpha-1 \text {. }
$$

\section{EXPERIMENT}

A set of measurements has been carried out to test the applicability of the results derived in the previous section. The laser used in these measurements is a vertical-jet cw unidirectional ring dye laser based on a design developed at JILA, University of Colorado, ${ }^{13}$ and the optical pump source for the dye laser is a Spectra-Physics 2016 argon-ion laser. The pump and signal power levels are measured with a Coherent 210 power meter, and the overall setup is shown schematically in Fig. 4. A polarization rotator is positioned between the pump and signal lasers, and the dye medium used in our experiments was a solution of rhodamine $6 \mathrm{G}$ in ethylene glycol at room temperature. The dye laser contains several Brewster-angle surfaces including the components of the Faraday isolator and the dye jet itself. No correction to the data has been made for the slight variation in pump intensity due to polarization-dependent reflection from the first surface of the dye jet, and this correction would be small compared to approximations made in the analysis.

A comparison of theoretical and experimental data is shown in Fig. 5. The theoretical curves in Fig. 5(a) are plots of the intensity from Eq. (25) as a function of the polarization rotation angle $\alpha$ for various values of the threshold parameter $r$. Basically, these curves are just an alternative way of representing the results shown already in Fig. 2. Figure 5(b) includes typical experimental data corresponding roughly to the same operating conditions. It
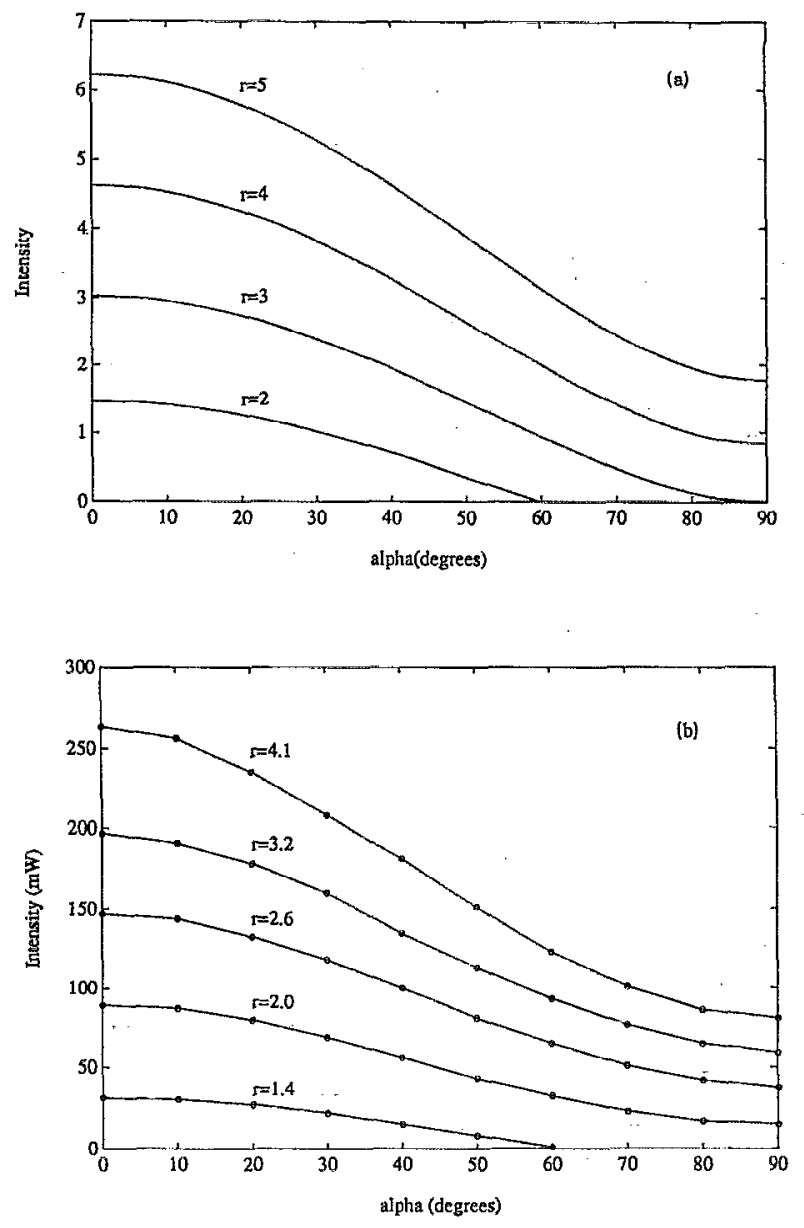

FIG. 5. Comparison of (a) the theoretical laser intensity and (b) the experimental intensity in milliwatt power units as a function of the polarization angle $\alpha$ of the pump beam in degrees at various levels of the threshold parameter $r$.

may be seen that there is good qualitative agreement between Figs. 5(a) and 5(b), but not so good quantitative agreement. In particular, we find that the dye laser reaches threshold for the perpendicular polarization direction for a threshold parameter of about $r=1.6$, whereas the theoretical model predicts a threshold value of $r=3$. On the other hand, the dye-laser output for both the parallel and perpendicular orientations is a quite linear function of the threshold parameter, and in this respect the model works well.

The significant discrepancy between the theoretical and experimental values of the threshold parameter in the perpendicular orientation is associated with the specific dye medium used in our studies. When rhodamine $6 \mathrm{G}$ molecules are suspended in a liquid solvent, their reorientation time is not long compared to the spontaneous decay time of the upper laser state. ${ }^{14-16}$ Hence, significant reorientation of the laser dipoles can occur between the time of excitation and stimulated emission. As a result, excited dipoles, which initially are almost perpendicular to the laser polarization, may still contribute significantly, and one result is a lowering of the laser threshold for the perpendicular pump configuration. A smaller correction which is not al- 
ways negligible in rhodamine $6 \mathrm{G}$ is a raising of the laser threshold due to intersystcm crossing into the triplet states. Both of these effects are medium dependent and can be reduced substantially by appropriate choice of the dye molecules and the material in which they are suspended.

In the absence of the signal saturation effects emphasized here, it has been possible to treat the threshold conditions for a dye laser including both rotational relaxation and intersystem crossing, ${ }^{7}$ and in the appropriate limits the results obtained are in exact agreement with the saturation formalism developed here. If follows from Eq. (32) that the angular dependence of the threshold parameter at the laser threshold can be written

$$
\begin{aligned}
r_{\text {th }} & =\frac{1}{1-\frac{2}{3} \sin ^{2} \alpha} \\
& =\frac{9}{\frac{3}{5}+\frac{6}{5} \cos ^{2} \alpha},
\end{aligned}
$$

where the second form is included for easier comparison with other results. On the other hand, it has been shown previously that when rotational diffusion and intersystem crossing to the triplet states are included, the threshold parameter is given approximately by Eq. (23) of Ref. 7:

$$
r_{\mathrm{th}}=\frac{\left(\frac{9}{5}+\tau_{2} / \tau_{r}\right)-K_{t}\left(1+\tau_{2} / \tau_{r}\right)}{\left(\frac{3}{5}+\frac{6}{5} \cos ^{2} \alpha+\tau_{2} / \tau_{r}\right)-K_{t}\left(1+\tau_{2} / \tau_{r}\right)},
$$

where $\tau_{r}$ is the rotational relaxation lifetime and $K_{t}$ is a normalized triplet-state parameter. This result obviously reduces to Eq. (37) in the limits $\tau_{r}>\tau_{2}$ and $K_{t}<1$. If the pump and signal polarizations are parallel, Eq. (38) becomes equal to unity, and if the pump and signal polarizations are perpendicular Eq. (38) takes on the value

$$
r_{\mathrm{th}}=\frac{\left(\frac{9}{5}+\tau_{2} / \tau_{r}\right)-K_{t}\left(1+\tau_{2} / \tau_{r}\right)}{\left(\frac{3}{5}+\tau_{2} / \tau_{r}\right)-K_{t}\left(1+\tau_{2} / \tau_{r}\right)},
$$

For rhodamine $6 \mathrm{G}$ dye, the triplet-state parameter is typically found to be on the order of $K_{t}=0.3 .^{7}$ The spontaneous relaxation time is usually estimated to be about 5 $\mathrm{ns},{ }^{14,15}$ and the rotational relaxation time in room-temperature ethylene glycol is about 2.2 ns. ${ }^{16}$ With these values, Eq. (39) yields $r_{\text {th }}=1.6$, and this value is in reasonable agreement with our experimental data shown in Fig. 5(b). Thus, our new model is not as accurate for threshold calculations of rhodamine $6 \mathrm{G}$ lasers with liquid solvents as compared to the best previous models, but unlike those models it includes arbitrary levels of signal saturation. For other intrinsically simpler lasers, it should provide quantitative accuracy.

\section{CONCLUSION}

Most practical dye-laser oscillators operate with Brewster elements in their optical cavities for low loss and, therefore, produce a linearly polarized output. Many of these lasers also operate with linearly polarized pump sources. Hence, it is of some interest to know how the performance of these lasers depends on the relative polarization alignment of the pump and signal fields. In this study general closed form results have been obtained for a dye laser having an isotropic distribution of dipole moments and an arbitrary angle between the pump and signal fields. These results are valid for any level of intensity. With a slight approximation, explicit formulas have been obtained for the intensity as a function of the polarization angle. Good qualitative agreement has been obtained with experimental data using a unidirectional rhodamine $6 \mathrm{G}$ ring dye laser. Quantitative agreement concerning the polarization alignment effects was not obtained primarily because of the orientational relaxation in the liquid-dye medium that we have used. Orientational relaxation is negligible in the synchronously pumped mode-locked dye lasers studied previously becausc of the very short time scale of the light-matter interactions, ${ }^{11}$ and this relaxation would, of course, also be negligible in solid-based dye lasers. Further studies of these polarization characteristics are in progress.

${ }^{1}$ P. P. Sorokin, J. R. Lankard, E. C. Hammond, and V. L. Moruzzi, IBM J. Res. Dev. 11, 130 (1967)

${ }^{2}$ A. A. Kovalev and V. A. Pilipovich, Bull. Acad. Sci. USSR, Phys. Ser. 34, 537 (1970).

${ }^{3}$ A. N. Sevchenko, A. A. Kovalev, and V. A. Pilipovich, Sov. Phys. Dokl. 14, 811 (1970).

${ }^{4}$ I. Nagata and T. Nakaya, J. Phys. D. 6, 1870 (1973).

${ }^{5}$ L. G. Pikulik and O. I. Yaroshenko, J. Appl. Spectrosc. 27, 861 (1977).

${ }^{6}$ L. G. Pikulik and O. I. Yaroshenko, J. Appl. Spectrosc. 27, 986 (1977)

${ }^{7}$ D. M. Phillion, D. J. Kuizenga, and A. E. Siegman, J. Chem. Phys. 61, 3828 (1974).

${ }^{8}$ K. C. Reyzer and L. W. Casperson, J. Appl. Phys. 52, 6075 (1980).

${ }^{2}$ K. C. Reyzer and L. W. Casperson, J. Appl. Phys. 52, 6083 (1980).

${ }^{10}$ L. I. Burov and I. I. Gancherenok, Opt. Spectrosc. (USSR) 61, 558 (1986).

${ }^{11}$ S. H. Jiang and L. W. Casperson, J. Appl. Phys. 69, 1866 (1991).

${ }^{12}$ L. W. Casperson, Appl. Opt. 19, 422 (1980).

${ }^{13} \mathrm{~J}$. L. Hall (private communication).

${ }^{14}$ A. Scavennec, Opt. Commun. 17, 14 (1976).

${ }^{15}$ C. P. Ausschnitt, R. K. Jain, and J. P. Heritage, IEEE J. Quantum Electron. QE-15, 912 (1979).

${ }^{16}$ T. J. Chuang and K. B. Eisenthal, Chem. Phys. Lett. 11, 368 (1971). 